\title{
ARITHMETICITY OF HOLONOMY GROUPS OF LIE FOLIATIONS
}

\author{
ROBERT J. ZIMMER
}

\section{INTRODUCTION}

The point of this paper is to prove arithmeticity of a class of finitely generated groups that arise naturally in a purely geometric context. Namely, we prove arithmeticity of the holonomy group of a Lie foliation of a compact manifold, assuming the existence of a metric for which the leaves are symmetric spaces of nonpositive curvature and rank at least 2 . This provides a partial answer to the question raised a number of years ago by A. Haefliger [13] of determining the possible holonomy groups of Lie foliations.

To indicate the nature of the questions we are considering, let us first recall that if $N$ is a Riemannian manifold, a fundamental question is to understand the relationship of the topology of $N$ and the geometry of $N$ (or for many purposes, the geometry of $\tilde{N}$ ). There has been a wide variety of work on this type of question from a variety of viewpoints. In particular, we can ask how the geometry of $N$ (or $\widetilde{N}$ ) controls the nature of $\pi_{1}(N)$. One can ask a more general question, namely to understand to what extent the geometry of $\widetilde{N}$ controls the actions of $\pi_{1}(N)$ on a manifold $T$. A standard construction associates to every such action a foliation of a manifold $M$ which has all leaves locally isometric to $\widetilde{N}$, in which $T$ appears naturally as a submanifold of $M$ transverse to the foliation, and the $\pi_{1}(N)$-action on $T$ controls the way in which the leaves of this foliation are "tied together". We can thus reinterpret our last question, and broaden its context as well, by formulating the following question: Determine the extent to which the geometry of a simply connected Riemannian manifold $X$ controls the way in which the leaves of a foliation are tied together if we assume the leaves are all locally isometric to $X$. In general, it is of course difficult to understand actions of a group on a manifold, and correspondingly difficult to understand foliations with leaves locally isometric to a given $X$. It is natural to begin an investigation of such actions with the case in which the action on $T$ preserves some geometric structure, e.g., a Riemannian metric. In terms of the associated foliation, this becomes a special case of the

Received by the editors October 26, 1986.

1980 Mathematics Subject Classification (1985 Revision). Primary 22E40, 28D15, 53C12, 57R30.

Research partially supported by NSF Grant DMS- 8301882 . 
notion of a "Riemannian foliation" (described in more detail below) in which the leaves are tied together in a way that preserves a metric. In this situation, generalizing the case of a foliation coming from an action of a fundamental group, there is a discrete group, called the holonomy group, with an embedding in a connected Lie group, that controls the way in which the leaves are tied together. The nature of the results we prove is that under certain assumptions on the geometry of $X$, this "tying together", i.e., the holonomy group together with an embedding in a connected Lie group, is of a very specific arithmetic nature.

To be more precise, we first recall the notion of a Lie foliation. Let $\mathscr{F}$ be a foliation of a compact connected manifold $M$. If $W \subset M$ is a sufficiently small open set, the foliation on $W$ is given by the fibers of a submersion $p: W \rightarrow U$. If $p_{i}: W_{i} \rightarrow U_{i}$ are two such submersions, we obtain a diffeomorphism (or homeomorphism if the foliation is only assumed to be continuous)

$$
f_{i j}: p_{i}\left(W_{i} \cap W_{j}\right) \rightarrow p_{j}\left(W_{i} \cap W_{j}\right) .
$$

If $X$ is a manifold and $G$ is an effective transformation group of $X$, we say that $\mathscr{F}$ is a $(G, X)$-foliation if we can choose a covering $\left\{W_{i}\right\}$ of $M$ such that $U_{i} \subset X$ is open and each $f_{i j}$ is the restriction of an element of $G$. This notion, and related notions, have been studied in [2, 7, 13, 21, 24], for example. In particular, if $X$ is a Riemannian manifold and $G$ is a group of isometries, $\mathscr{F}$ is called a Riemannian foliation, and if $X=G$, a connected Lie group, then $\mathscr{F}$ is called a Lie foliation, or more precisely, a $G$-foliation. These cases have been studied in $[4,8,9,12,17,22]$, and elsewhere. By passing to a transverse orthogonal frame bundle, the study of Riemannian foliations can in most respects be reduced to the study of Lie foliations (see, e.g., [17]). It is clear that if $G$ and $G^{\prime}$ are locally isomorphic, a $G$-foliation is also a $G^{\prime}$-foliation. Thus, one can speak more properly of a $\mathfrak{g}$-foliation, where $\mathfrak{g}$ is a Lie algebra.

For any $G$-foliation, there is a natural homomorphism $h: \pi_{1}(M) \rightarrow G$, the holonomy homomorphism, and a locally trivial fibration $D: \widetilde{M} \rightarrow G$ (the developing map) which commutes with the action of $\pi_{1}(M)$ (where $\pi_{1}(M)$ acts on $G$ via translation by $\left.h(\gamma), \gamma \in \pi_{1}(M)\right)$. Here $\widetilde{M}$ is the covering of $M$ corresponding to the normal subgroup $\operatorname{ker}(h)$. Furthermore, the leaves of $\mathscr{F}$ are exactly the images of the fibers of $D$ under the covering projection $\widetilde{M} \rightarrow M$. The group $h\left(\pi_{1}(M)\right)=\Gamma \subset G$ is called the holonomy group of the $G$-foliation. Various general results on Lie foliations allow reduction to the case of foliations with a dense leaf [17]. In this case, the holonomy group $\Gamma$ will be dense in $G$. For the construction of $h$ and $D$, see [8, 24]. If the fibers of $D$ are connected, then the leaves of $\mathscr{F}$ are in natural 1-1 correspondence with the $\Gamma$-orbits in $G$. We then say that $\mathscr{F}$ has good development over $G$. If $G$ is simply connected, this will always be the case, but it may or may not be true for other groups locally isomorphic to $G$.

Suppose now that $G$ is a connected semisimple Lie group with finite center and that $\Gamma \subset G$ is a dense subgroup. Then $\Gamma$ is called arithmetic if there is a 
semisimple algebraic Q-group $H$ and a smooth surjection $p: H_{\mathbf{R}}^{0} \rightarrow \operatorname{Ad}_{G}(G)$ such that $p\left(H_{\mathbf{R}}^{0} \cap H_{\mathbf{z}}\right)$ and $\operatorname{Ad}_{G}(\Gamma)$ are commensurable (cf. the definition of arithmeticity for discrete subgroups [28]). In light of Margulis' arithmeticity theorem $[15,28]$, this is equivalent to the condition that there is a semisimple Lie group $G^{\prime}$ and an irreducible lattice $\Lambda \subset \operatorname{Ad}_{G}(G) \times G^{\prime}$ such that $\operatorname{Ad}_{G}(\Gamma)$ is commensurable with the projection of $\Lambda$ into $\operatorname{Ad}_{G}(G)$. While this latter formulation is perhaps more natural in a geometric context (although it carries less information without Margulis' theorem), we wish to emphasize the arithmetic formulation here. This is because we shall show that a suitable holonomy group is arithmetic not by first showing it is a lattice and then applying Margulis' theorem, but rather by showing via other methods that it is naturally a subgroup of a group of the form $H_{\mathbf{z}}$ where $H$ is a Q-group and then using further arguments to show that it must be of finite index in this group. Thus, in our proof, the fact that the group is a lattice will follow after we establish its arithmetic nature, rather than vice versa by an application of Margulis' theorem.

We recall that any symmetric space $Y$ of nonpositive curvature has a (essentially unique) de Rham decomposition $Y=\Pi Y_{i}$, where each $Y_{i}$ is an irreducible symmetric space of nonpositive curvature. We let

$$
d(Y)=\min \left\{\operatorname{dim} \operatorname{Isom}\left(Y_{i}\right)\right\}
$$

where $\operatorname{Isom}\left(Y_{i}\right)$ is the group of isometries of $Y_{i}$ (and is a noncompact simple Lie group if $Y_{i}$ is not Euclidean).

We can now formulate a precise version of our main results.

Theorem A. Let $\mathfrak{g}$ be a (real, finite dimensional) Lie algebra and $\mathscr{F}$ a $\mathfrak{g}$-foliation of a compact connected manifold $M$. Suppose there is a dense, simply connected leaf. Assume there is a Riemannian metric on $M$ such that each leaf is a locally symmetric space of nonpositive curvature such that all irreducible factors in the de Rham decomposition of the covering symmetric space $Y$ have rank at least 2 . Then:

(1) $\mathfrak{g}$ is semisimple.

(2) There is a (connected) Lie group $G$ with finite center (and Lie algebra $\mathfrak{g})$ such that $\mathscr{F}$ has good development over $G$.

(3) The holonomy group $\Gamma \subset G$ is a dense arithmetic subgroup.

(4) $\operatorname{Ad}_{G}(\Gamma)$ is commensurable with the projection into $\operatorname{Ad}_{G}(G)$ of a lattice in $\operatorname{Ad}_{G}(G) \times \operatorname{Isom}(Y)$.

(5) $\operatorname{codim}(\mathscr{F})=\operatorname{dim} \mathfrak{g} \geq d(Y)$.

As indicated above, the hypothesis that $\mathscr{F}$ has a dense leaf is not a serious restriction. It is possible that Theorem $A$ remains true without the assumption that there is such a leaf which is simply connected. In this direction, our arguments show:

Theorem B. Assume all hypotheses of Theorem A, except the hypothesis that there is a simply connected leaf. (We still assume there is a dense leaf.) Then $\operatorname{codim} \mathscr{F} \geq d(Y) / 2$. 
Theorem B implies of course the nonexistence in low codimension of Lie foliations with a dense leaf and leaves locally isometric to $Y$. Via the reduction of Riemannian foliations to Lie foliations alluded to above (see [17]), we obtain the following consequence.

Corollary C. Let $\mathscr{F}$ be a Riemannian foliation of a compact manifold $M$ with codimension $d$. Assume there is a metric on $M$ such that the leaves of $\mathscr{F}$ are locally isometric to a symmetric space $Y$ all of whose irreducible factors have rank at least 2. If $d(d+1)<d(Y)$, then all leaves are compact. Moreover, there is a finite covering $\widetilde{M}$ of $M$ such that the lifted foliation $\widetilde{F}$ on $\widetilde{M}$ is the foliation defined by a fiber bundle projection.

A priori, the holonomy group of a Lie foliation is a finitely generated subgroup of a Lie group. Haefliger [13] raised the general problem of understanding which such groups may arise this way. The general nature of Theorem A is of course that it is a theorem relating the geometry of the leaves to the holonomy embedding $\Gamma \hookrightarrow G$. There are other such results. Namely, in [4] it is shown that for a Lie algebra $\mathfrak{g}, \mathfrak{a} \mathfrak{g}$-foliation has leaves of polynomial growth if and only if $\mathfrak{g}$ is nilpotent, and that the leaves are "Fölner" (i.e. satisfy a certain isoperimetric inequality) if and only if $\mathfrak{g}$ is solvable. In [8], $\left(\mathrm{O}(1, n), H^{n}\right)$ foliations $\left(H^{n}=\right.$ hyperbolic $n$ space) are studied in case $\operatorname{dim} \mathscr{F}=1$, i.e. the leaves are locally isometric to the line.

As we remarked above, our proof of Theorem A does not employ Margulis' arithmeticity theorem, but the proofs do have certain features in common. Namely, the basic step in the proof of Margulis' theorem is his "superrigidity" theorem. In [26] (see also [27, 28]) we generalized Margulis' superrigidity theorem to obtain "superrigidity for cocycles", a result which gives detailed measure-theoretic information about actions of semisimple groups on vector (or principal) bundles. This theorem will in turn play a basic role in the proof of the present arithmeticity theorem (i.e. Theorem A).

Although the hypotheses of Theorem A are of a geometric and topological nature, the more difficult and central part of the argument in the proof involves the relationship of algebraic groups and ergodic theory. In fact, we shall basically deduce Theorem A from the following result in ergodic theory. We refer the reader to $[10,11]$ and $\S 3$ below for the notion of measurable stable orbit equivalence.

Theorem D. Let $G$ be a connected Lie group and $\Gamma \subset G$ a dense finitely generated subgroup. Let $H$ be a connected semisimple adjoint Lie group, each of whose simple factors has $\mathbf{R}$-rank at least 2 . Suppose the action of $\Gamma$ on $G$ (by translations) is measurably stably orbit equivalent to an essentially free action of $H$ on a (standard) measure space preserving a finite measure. Then:

(1) $G$ is semisimple.

(2) $\Gamma \cap Z(G)$ is of finite index in $Z(G)$.

(3) $\operatorname{Ad}_{G}(\Gamma)$ is a dense arithmetic subgroup of $\operatorname{Ad}_{G}(G)$. 
(4) $\operatorname{Ad}_{G}(\Gamma)$ is commensurable with the projection into $\operatorname{Ad}_{G}(G)$ of a lattice in $\operatorname{Ad}_{G}(G) \times H$.

We remark that the properties of the measurable equivalence relation defined by the action of a dense subgroup on a Lie group also play a role in the results of [4] described above on $\mathfrak{g}$-foliations with $\mathfrak{g}$ solvable. Namely, in [31] we showed that if $\Gamma$ is a dense subgroup of $G$, where $G$ is a connected Lie group, then the $\Gamma$ action on $G$ is amenable if and only if $G$ is solvable. This was applied in [4] in the proof that for a $\mathfrak{g}$-foliation, the leaves are Fölner if and only if $\mathfrak{g}$ is solvable.

$\S \S 2-10$ of this paper are devoted to the proof of the results stated above. In $\S 11$, we show how one can obtain results similar to Corollary $C$ for foliations in which the leaves are locally isometric to certain rank one symmetric spaces of negative curvature, namely those whose isometry group is a Kazhdan group. This is based on a result proved in $\S 11$, concerning stable orbit equivalence of the action defined by a dense subgroup of a Lie group with a finite measure preserving action of a Kazhdan group.

I would like to thank Y. Carriere and E. Ghys for communicating their papers $[4,5]$ which stimulated my interest in some of these matters.

\section{Preliminaries on foliations}

We recall from the introduction the following definition.

Definition 2.1. Let $\mathfrak{g}$ be a (real, finite dimensional) Lie algebra and $G$ a connected group with Lie algebra $\mathfrak{g}$. Let $\mathscr{F}$ be a $\mathfrak{g}$-foliation of a compact, connected manifold $M, h: \pi_{1}(M) \rightarrow G$ the holonomy homomorphism, and $D: \widetilde{M} \rightarrow G$ the developing map, where $\widetilde{M}$ is the covering of $M$ corresponding to $\operatorname{ker}(h)$. We say that $\mathscr{F}$ has good development over $G$ if the fibers of $D$ are connected.

We then have the following easily verified assertions.

Proposition 2.2. (a) If $G_{1} \rightarrow G_{2}$ is a covering and $\mathscr{F}$ has good development over $G_{2}$, it has good development over $G_{1}$.

(b) If $G$ is simply connected, $\mathscr{F}$ has good development over $G$.

We let $M / \mathscr{F}$ denote the (in general non-Hausdorff) space of leaves.

Proposition 2.3. If $\mathscr{F}$ has good development over $G$, with $h\left(\pi_{1}(M)\right)=\Gamma$, then the $\pi_{1}(M)$-map $D: \widetilde{M} \rightarrow G$ induces a bijection of $M / \mathscr{F}$ with $G / \Gamma$.

(We remark that in general, $\Gamma$ is not closed in $G$.)

It will be convenient to express this bijection in an alternate manner.

Proposition 2.4. If $\mathscr{F}$ has good development over $G$, then there is a Borel isomorphism $\theta$ of $G$ with a Borel subset $T \subset M$ such that:

(a) $T$ intersects every leaf at least once, and in at most a countable set. 
(b) If $x, y \in T$, then $x$ and $y$ are in the same leaf if and only if $\theta^{-1} x$ and $\theta^{-1} y$ are in the same $\Gamma$-orbit in $G$.

Proof. We can choose a Borel section of the $\Gamma$-map $D: \widetilde{M} \rightarrow G$, and with some care the image of this section will map injectively under projection to $M$. Let $T$ be this image in $M$.

We shall need the following observation about foliations by locally symmetric spaces.

Proposition 2.5. Let $\mathscr{F}$ be a foliation of a compact manifold $M$. Assume each leaf is locally isometric to a symmetric space $Y$ of nonpositive curvature. Let $H$ be the connected component of the identity of the isometry group of $Y$. Fix $y_{0} \in Y$ and let $K$ be the stabilizer of $y_{0}$ in $H$, so that $K$ is a compact Lie group. Then there is a principal $K$-bundle $\rho: M^{*} \rightarrow M$ with a smooth $H$-action on $M^{*}$ such that the $H$-orbits in $M^{*}$ are precisely the inverse images under $\rho$ of the leaves of $\mathscr{F}$. Further, for $x \in M^{*}$, the stabilizer $H_{x}$ is isomorphic to the fundamental group of the leaf of $\mathscr{F}$ through $\rho(x)$. If $\mathscr{F}$ is a Riemannian or Lie foliation (or more generally if there is a transverse invariant measure of smooth class) then there is a finite $H$-invariant measure on $M$ of smooth class.

Proof. Let $M^{*}$ be the set of smooth maps $q: Y \rightarrow M$ such that $q$ is a Riemannian covering of a leaf of $\mathscr{F}$. If $h \in H$, clearly $q \circ h \in M^{*}$, so that $H$ acts on $M^{*}$. Fix a point $y_{0} \in Y$ and define $\rho: M^{*} \rightarrow M$ by $\rho(q)=q\left(y_{0}\right)$. Then all assertions of the proposition may be verified in a routine manner.

\section{Preliminaries on ergodic theory}

In this section we review a number of ergodic theoretic notions that we will need for the proofs of the main theorems. There will be a number of statements in which equality should be interpreted as equality almost everywhere. We may occasionally be careful in this direction, but for the most part this distinction causes only routine problems and we will usually ignore them. In subsequent sections there will be a significant number of known results we shall use freely, providing references but not in general discussing them as background material. The reader is referred to [28] for a general discussion of the ideas involved.

We begin by recalling the notion of orbit equivalence and stable orbit equivalence. We refer the reader to $[10,11,20,27,28]$ and the references therein for more detail. Suppose $G$ and $H$ are locally compact groups acting on (standard) measure spaces $(X, \mu)$ and $(Y, \nu)$ respectively. The measures $\mu$ and $\nu$ are assumed to be $\sigma$-finite and quasi-invariant. The actions are called orbit equivalent if (possibly after discarding null sets) there is a measurable and measure class preserving bijection $\theta: X \rightarrow Y$ such that $\theta$ takes $G$-orbits onto $H$-orbits; more precisely, for (a.e.) $x \in X, \theta(x G)=\theta(x) H$. A somewhat weaker notion, whose measure theoretic properties were examined in detail in 
$[10,11,20]$ (although in a slightly different context in [20]) is the notion of stable orbit equivalence.

Proposition 3.1 [11]. The following are equivalent.

(i) The action of $G \times S^{1}$ on $X \times S^{1}$ is orbit equivalent to the action of $H \times S^{1}$ on $Y \times S^{1}$ (where $S^{1}$ is the circle).

(ii) After discarding invariant null sets, there is a measurable map $\theta: X \rightarrow Y$ such that $\theta$ induces a null set preserving bijection $X / G \rightarrow Y / H$. (We remark that we do not assume $\theta$ itself to be either injective or surjective.)

Definition 3.2. If the conclusions of 3.1 are satisfied, the $G$-action on $X$ and the $H$-action on $Y$ are called stably orbit equivalent.

We say that an action has "continuous orbits" if (almost) every orbit is uncountable.

Proposition 3.3 [11]. (a) If two actions are stably orbit equivalent, one is ergodic if and only if the other is ergodic.

(b) Actions with continuous orbits are stably orbit equivalent if and only if they are orbit equivalent.

(c) If the $G$-action on $X$ has continuous orbits and $H$ is a countable group acting on $Y$, then the actions are stably orbit equivalent if and only if (after discarding invariant null sets) there is a Borel isomorphism $\theta$ of $Y$ with a Borel subset $T \subset X$ such that $\theta$ induces a null set preserving bijection $Y / H \rightarrow X / G$.

Example 3.4. Suppose $\mathscr{F}$ is a foliation of a compact manifold $M$ and that the leaves are locally homogeneous spaces with a common universal cover $\widetilde{L}$. Let $H$ be the connected component of the identity of the isometry group of $\widetilde{L}$. Suppose $\mathscr{F}$ is a $\mathfrak{g}$-foliation with good development over $G$. Let $\Gamma \subset G$ be the holonomy group. Then the action of $H$ on $M^{*}$ (where $M^{*}$ is as in Proposition 2.5 ) is stably orbit equivalent to the $\Gamma$-action on $G$.

Proof. This follows from 2.4, its proof, 2.5, and 3.3.

A basic property of stable orbit equivalence is that it induces isomorphism on cohomology. Before stating this in the form we need, we recall some facts about cocycles (see [28] as a general reference).

We recall that if $G$ acts on (the right of) $X$ and $L$ is a locally compact group, a Borel function $\alpha: X \times G \rightarrow L$ is called a cocycle if for all $g, h \in$ $G, \alpha(x, g h)=\alpha(x, g) \alpha(x g, h)$ (a.e. $x)$. Two such cocycles $\alpha, \beta$ are called equivalent or cohomologous (and we write $\alpha \sim \beta$ ) if there is a measurable $\phi: X \rightarrow L$ such that for each $g \in G, \phi(x) \alpha(x, g) \phi(x g)^{-1}=\beta(x, g)$ (a.e.). We denote by $Z^{1}((X, G) ; L)$ the space of cocycles and by $H^{1}((X, G) ; L)$ the set of cohomology classes of cocycles. If $L_{1} \subset L$ is a subgroup, it is often of interest to know when $\alpha$ is cohomologous to a cocycle taking all values in $L_{1}$. If $Y$ is an $L$-space, a function $\phi: X \rightarrow Y$ is called $\alpha$-invariant if $\phi(x) \alpha(x, g)=\phi(x g)$. The following is basically formal. 
Proposition 3.5. The following are equivalent:

(i) $\alpha$ is equivalent to a cocycle taking all values in $L_{1}$;

(ii) there is a measurable $\alpha$-invariant $\phi: X \rightarrow L / L_{1}$.

Using this, one can define the important notion of the algebraic hull of a cocycle taking values in an algebraic group. (See $[28,30]$ for a proof and discussion of the following result.)

Proposition 3.6. Let $k$ be a local field of characteristic $0, L$ an algebraic $k$-group, and $L_{k}$ the group of $k$-points. Suppose $G$ is a locally compact group acting ergodically on $X$, and that $\alpha: X \times G \rightarrow L_{k}$ is a cocycle. Then there is an algebraic $k$-group $H \subset L$ with the following properties.

(i) $\alpha$ is equivalent to a cocycle taking all its values in $H_{k}$.

(ii) For any algebraic $k$-group $H^{\prime}$ with $H_{k}^{\prime} \subset H_{k}$ a proper inclusion, $\alpha$ is not equivalent to a cocycle taking all its values in $H_{k}^{\prime}$.

(iii) Up to conjugacy in $L_{k}, H_{k}$ is the unique such group satisfying (i), (ii).

(iv) If $\alpha$ is equivalent to a cocycle taking all its values in some algebraic group $H_{k}^{\prime} \subset L_{k}$, then some conjugate of $H_{k}$ in $L_{k}$ is contained in $H_{k}^{\prime}$.

Definition 3.7 [28]. The group $H_{k}$ (or more precisely its conjugacy class in $L_{k}$ ) is called the algebraic hull of $\alpha$. The algebraic hull is an invariant of the cohomology class defined by $\alpha$. We say that $\alpha$ is Zariski dense in $L_{k}$ if the algebraic hull is $L_{k}$ itself.

Example 3.8 [28]. Suppose $G$ is a semisimple connected real algebraic group with no compact factors, and $X$ is a $G$-space on which $G$ acts ergodically with finite invariant measure. Let $\alpha(x, g)=g$, so that $\alpha: X \times G \rightarrow G$ is a cocycle. If $H$ is a closed subgroup, then $\alpha$ is equivalent to a cocycle taking all values in $H$ if and only if there is a $G$-map $X \rightarrow G / H$. If $H$ is algebraic, the Borel density theorem $[3,28]$ implies $H=G$. Thus, $\alpha$ is Zariski dense in $G$.

In general, an ergodic action of a real algebraic group $G$ is called Zariski dense in $G$ if the cocycle $\alpha(x, g)=g$ is Zariski dense. This is equivalent to the assertion that there is no measurable $G$-map $X \rightarrow G / H$ where $H \subset G$ is proper algebraic.

A cocycle $\alpha: X \times G \rightarrow L$ is called orbital if for (almost) all $x \in X$, $\alpha(x, g)=e$ for all $g \in G_{x}$, the latter being the stablizer of $x$ in $G$. If $\alpha \sim \beta$, then $\alpha$ is orbital if and only if $\beta$ is orbital. We let $Z_{\text {orb }}^{1}((X, G) ; L)$ (resp. $\left.H_{\text {orb }}^{1}((X, G) ; L)\right)$ denote the set of (resp., equivalence classes of) orbital cocycles.

Lemma 3.9. Suppose $G$ acts on $(X, \mu)$. Let $p: X \times S^{1} \rightarrow X$ be projection. Then the map

$$
p^{*}: Z_{\text {orb }}^{1}((X, G) ; L) \rightarrow Z_{\text {orb }}^{1}\left(\left(X \times S^{1}, G \times S^{1}\right) ; L\right)
$$


given by $\left(p^{*} \alpha\right)((x, s),(g, z))=\alpha(x, g)$ induces a bijection on orbital cohomology. The inverse is the map on cohomology induced by $i: X \rightarrow X \times S^{1}$, $i(x)=(x, e)$, i.e. is given by $\left(i^{*} \beta\right)(x, g)=\beta((x, e),(g, e))$. If the $G$-action on $X$ is ergodic, and $L$ is (the $k$-points of ) an algebraic group, then the algebraic hull of $\alpha$ is equal to the algebraic hull of $p^{*} \alpha$.

Proof. All but the last assertion follows from [11, 20]. The last assertion is easily verified.

Corollary 3.10. If $(X, G)$ and $(Y, H)$ are stably orbit equivalent ergodic actions, then there is a bijection $H_{\mathrm{orb}}^{1}((X, G) ; L) \rightarrow H_{\mathrm{orb}}^{1}((Y, H) ; L)$ preserving algebraic hulls.

Example 3.11. Consider the situation in Proposition 3.3(c). Suppose further that the $G$-action on $X$ is ergodic and essentially free (i.e. almost all stabilizers are trivial). We then have a cocycle $\alpha: Y \times H \rightarrow G$ characterized by $\theta(y) \alpha(y, h)=\theta(y h)$ (cf. [28, 4.2.8]). Under the isomorphism of Corollary 3.10 , this corresponds to the cocycle $\beta: X \times G \rightarrow G$ given by $\beta(x, g)=g$. In particular, if $G$ is semisimple with no compact factors, and the measure on $X$ is finite and invariant, then $\alpha$ is Zariski dense in $G$ by Example 3.8.

If $G$ is a connected semisimple Lie group for which all simple factors have R-rank at least 2 , then we have very explicit information on the cocycles with a simple algebraic hull. If $\pi: G \rightarrow L$ is a continuous homomorphism, let $\alpha_{\pi}(x, g)=\pi(g)$, so that $\alpha_{\pi}: X \times G \rightarrow L$ is a cocycle. The following result is fundamental for the proofs of Theorems A, B, and D.

Theorem 3.12 [26, 27, 28] (Superrigidity for cocycles). Let $G$ be a connected semisimple Lie group with finite center such that every simple factor of $G$ has R-rank at least 2 . Let $(X, \mu)$ be an ergodic $G$-space with finite invariant measure. Let $\alpha: X \times G \rightarrow L$ be a cocycle.

(a) If $L$ is a simple adjoint noncompact Lie group and $\alpha$ is Zariski dense in $L$, then there is a (smooth) homomorphism $\pi: G \rightarrow L$ (necessarily surjective) such that $\alpha \sim \alpha_{\pi}$.

(b) If $L$ is a simple adjoint complex algebraic group, and $\alpha$ is Zariski dense in $L$, then either: (i) there is a smooth homomorphism $\pi: G \rightarrow L$ such that $\alpha \sim \alpha_{\pi}$; or (ii) $\alpha \sim \beta$ where $\beta$ takes all values in a compact subgroup of $L$.

(c) If $L$ is a group of $k$-points of a $k$-group where $k$ is a totally disconnected local field of characteristic 0 , then $\alpha \sim \beta$ where $\beta$ takes all values in a compact subgroup of $L$.

This result is a generalization of Margulis' superrigidity theorem [15, 28]. Theorem 3.12 was first proven under slightly different hypotheses in [26] (see $[27,28]$ for the present hypotheses), where it was used to prove results about orbit equivalence of actions of semisimple groups. Here we shall need the following variant of the application given in [26]. 
Corollary 3.13. Let $G$ and $X$ be as in 3.12 (and assume the $G$-action on $X$ is effective). Let $H$ be a connected semisimple adjoint Lie group with no compact factors, and $Y$ an ergodic $H$-space which is essentially free. Suppose further that the action on $Y$ is Zariski dense in $H$. (We do not assume, however, that the $H$-action has a finite invariant measure.) Suppose the $G$-action on $X$ and $H$-action on $Y$ are stably orbit equivalent. Then there is a surjective (smooth) local isomorphism $\pi: G \rightarrow H$ and a measure class preserving map $\psi: X \rightarrow Y$ such that $\psi(x g)=\psi(x) \pi(g)$. In particular, $Y$ has a finite $H$-invariant measure (in the given measure class).

Proof. This follows from the proof of [28, Theorem 5.2.1]. In this proof, only the Zariski density of the action of $H$ and $Y$ is used, not the fact that there is a finite invariant measure.

Another very useful result about cocycles for such $G$ derives from the fact that $G$ has Kazhdan's property.

Theorem 3.14 [28, Theorem 9.1.1]. Assume $G$ is a locally compact Kazhdan group (e.g., $G$ is a connected semisimple Lie group with finite center for which every simple factor has $\mathbf{R}$-rank at least 2 ). Let $X$ be an ergodic $G$-space with a finite invariant measure, and $\alpha: X \times G \rightarrow L$ a cocycle, where $L$ is an amenable locally compact group. Then $\alpha$ is equivalent to a cocycle taking all values in $a$ compact subgroup of $L$.

We can now indicate the relevance of these considerations for the proof of Theorems A and B. By Example 3.4, the holonomy action of $\Gamma$ on $G$ is stably orbit equivalent to a finite measure preserving ergodic action of a semisimple Lie group, each of whose simple factors has R-rank at least 2. Theorems 3.12 and 3.14 give us some control over the cohomology of the latter action and by Corollary 3.10, we have information on $H^{1}$ for the $\Gamma$-action on $G$. Each homomorphism of $\Gamma$ in turn defines an element of $H^{1}$, and thus we can hope to derive information about homomorphisms of $\Gamma$. This, of course, has the potential of leading one to an arithmeticity theorem.

\section{A HOMOMORPHISM EXTENSION THEOREM}

In this section we describe a basic result on the extension of homomorphisms of dense subgroups to homomorphisms of the ambient group. The technique is basically due to Margulis and appears in approximately the following form in our discussion of Margulis' work in [28].

Theorem 4.1. Let $\Lambda$ be a dense subgroup of a locally compact group L. Let $k$ be a local field of characteristic $0, M$ an (algebraically) connected $k$-group and $\pi: \Lambda \rightarrow M_{k}$ a homomorphism with $\pi(\Lambda)$ Zariski dense. Suppose there is a measurable $\Lambda$-map $\phi: L \rightarrow M_{k} / N_{k}$ where $N \subset M$ is a $k$-subgroup for which $M_{k}$ acts effectively on $M_{k} / N_{k}$. Then $\pi$ extends to a continuous homomorphism $L \rightarrow M_{k}$. 
Proof. This is implicit in the argument of [28, p. 128] but we shall include some details for completeness.

Let $F\left(L, M_{k} / N_{k}\right)$ denote the space of measurable maps $\phi: L \rightarrow M_{k} / N_{k}$, two such maps being identified if they agree almost everywhere. This space has the topology of convergence in measure on compact sets. The group $M_{k}$ acts on $F\left(L, M_{k} / N_{k}\right)$ pointwise in the image, and $L$ acts on this space by translation on the domain. Since $\Lambda \subset L$ is dense and $M_{k}$ and $N_{k}$ are algebraic, we can apply the argument of $\left[28\right.$, p. 128]. This will show that there is a point $x_{0} \in M_{k} / N_{k}$ and a measurable (and hence continuous) homomorphism $\sigma: L \rightarrow M_{k}$ such that for $h \in L, \phi(h)=x_{0} \cdot \sigma(h)$, provided we can establish that $\left\{m \in M_{k} \mid x\right.$. $m=x$ for all $x \in \phi(L)\}$ is trivial. However, $\phi(L) \cdot \pi(\Lambda) \subset \phi(L)$ since $\phi$ is a $\Lambda$-map and hence the Zariski closure of $\phi(L)$ is $\pi(\Lambda)$-invariant (more precisely, the Zariski closure of the essential range of $\phi)$. Since $\pi(\Lambda)$ is Zariski dense in $M$, this implies $\phi(L)$ is Zariski dense. Thus, if $m \in M_{k}$ pointwise fixes $\phi(L)$, it pointwise fixes $M_{k} / N_{k}$ which by our effectiveness assumption implies $m$ is trivial. To complete the proof, it suffices to show that $\sigma$ extends $\pi$. For $\gamma \in \Lambda$ and (a.e.) $h \in L$, we have

$$
\phi(h \gamma)=x_{0} \cdot \sigma(h \gamma)=x_{0} \cdot \sigma(h) \sigma(\gamma)=\phi(h) \sigma(\gamma),
$$

and $\phi(h \gamma)=\phi(h) \pi(\gamma)$. Therefore, for almost all $h$ we have $\phi(h) \sigma(\gamma)=$ $\phi(h) \pi(\gamma)$. Therefore $\sigma(\gamma) \pi(\gamma)^{-1}$ pointwise fixes the essential range of $\phi$. As we remarked above, this set is Zariski dense, so $\sigma(\gamma) \pi(\gamma)^{-1}$ acts trivially on $M_{k} / N_{k}$, showing $\sigma(\gamma)=\pi(\gamma)$.

Suppose now that $G$ is a connected Lie group. Then there is a unique maximal solvable normal subgroup $R \subset G$ such that $G / R$ is a connected adjoint semisimple Lie group. Namely, let $p: G \rightarrow G / \operatorname{rad}(G)$ be the natural projection where $\operatorname{rad}(G)$ is the radical. Then let $R=p^{-1}(Z(G / \operatorname{rad}(G)))$. We then have the following corollary of Theorem 4.1 .

Corollary 4.2. Let $G$ be a connected Lie group, $R$ as above, $p: G \rightarrow G / R$. Let $\Gamma \subset G$ be a dense subgroup, and let $M$ be a connected semisimple adjoint real or complex algebraic group. Suppose $N \subset M$ is an algebraic subgroup not containing a nontrivial normal subgroup of $M$. Let $\pi: \Gamma \rightarrow M$ be a homomorphism with $\pi(\Gamma)$ Zariski dense. If there is a measurable $\Gamma$-map $\phi: G \rightarrow M / N$, then there is a rational homomorphism $\sigma: G / R \rightarrow M$ such that $\pi=\sigma \circ(p \mid \Gamma)$.

Proof. By Theorem 4.1, $\pi$ extends to a continuous homomorphism $\sigma: G \rightarrow$ $M$, and since $\pi(\Lambda)$ is Zariski dense in $M$ and $M$ is semisimple and adjoint, $\sigma \mid R$ is trivial.

For totally disconnected groups we obtain:

Corollary 4.3. Let $G$ be a connected Lie group, and $\Gamma \subset G$ a dense subgroup. Let $k$ be a totally disconnected local field of characteristic 0 , and $M$ an (algebraically) connected simple adjoint k-group. Suppose $\pi: \Gamma \rightarrow M_{k}$ is 
a homomorphism with $\pi(\Gamma)$ Zariski dense. If $N \subset M$ is a proper $k$-subgroup, then there is no measurable $\Gamma$-map $G \rightarrow M_{k} / N_{k}$.

\section{First RESULTS ON $R$}

We now begin the proof of Theorems A, B, and D. To fix notation, we assume $G$ is a connected Lie group, and $\Gamma \subset G$ a countable dense subgroup. Let $\operatorname{rad}(G)$ be the radical and $R \supset \operatorname{rad}(G)$ the maximal solvable normal subgroup with $G / R=L$ a connected semisimple adjoint Lie group (see $\S 4$ ). While $R$ is solvable, it is not necessarily connected. We let $H$ be a connected semisimple adjoint Lie group each of whose simple factors has R-rank at least 2 . Let $X$ be an ergodic, essentially locally free (i.e. almost all stabilizers are discrete) $H$-space with a finite invariant measure. We assume the actions of $H$ on $X$ and $\Gamma$ on $G$ are stably orbit equivalent. In this section we obtain some first results on $R$ and $\Gamma \cap R$. We shall of course subsequently obtain much sharper information.

Lemma 5.1. $R \neq G$, i.e. $L$ is nontrivial.

Proof. Suppose $R=G$. Since $R$ is solvable, the action of $\Gamma$ on $R$ is amenable. It follows that there is a cocycle $R \times \Gamma \rightarrow \mathbf{R}$ whose algebraic hull is $\mathbf{R}$. (This can be deduced from [6], which shows that an amenable action is orbit equivalent to a $\mathbf{Z}$-action, and the construction of cocycles on $\mathbf{Z}$-actions in [23]. From the latter, one obtains a cocycle $\alpha$ for which the skew product action of $\Gamma$ on $R \times \mathbf{R}$, given by $(r, t) \cdot \gamma=(r \gamma, t+\alpha(r, \gamma))$ is ergodic. This easily implies that the algebraic hull is $\mathbf{R}$.) By Corollary 3.10 , there is a cocycle $\alpha: X \times H \rightarrow \mathbf{R}$ with algebraic hull $\mathbf{R}$. However, this contradicts Theorem 3.14.

Remark. The proof shows that the lemma is valid if $H$ is any Kazhdan group, not necessarily a semisimple group with all simple factors of R-rank at least 2 .

The next result we establish under the additional assumption that the $H$-action is essentially free.

Lemma 5.2. Assume the $H$-action on $X$ is essentially free. Then $\Gamma \cap R$ is discrete.

Remark. This argument will apply for $H$ any semisimple Lie group with no compact factors.

Proof. Let $R_{1}=(\overline{\Gamma \cap R})$. Since $R$ is normal in $G, \Gamma$ normalizes $R_{1}$, and since $\Gamma$ is dense in $G, R_{1} \subset G$ is normal. Let $E=G / R_{1}$ and $q: G \rightarrow E$ be the natural map. We remark that $R_{1}$ is solvable and hence that $\Gamma \cap R$ is an amenable dense subgroup of $R_{1}$.

Let $\alpha: G \times \Gamma \rightarrow H$ be the cocycle given by Example 3.11. Thus, we choose a Borel isomorphism $\theta: G \rightarrow T \subset X$ and define $\alpha$ by $\theta(g \gamma)=\theta(g) \alpha(g, \gamma)$. (The existence of $\alpha$ depends upon the assumption that the $H$-action is essentially free.) By the remarks in Example 3.11, $\alpha$ is Zariski dense in $H$. Let 
$\alpha_{1}=\alpha \mid G \times(\Gamma \cap R)$. Let $P \subset H$ be a minimal parabolic subgroup, and $M(H / P)$ the space of probability measures on $H / P$ with the weak- ${ }^{*}$-topology. (We recall $H / P$ is compact, and hence $M(H / P)$ is as well.) Since $\Gamma \cap R$ is amenable, the action of $\Gamma \cap R$ on $G$ is amenable [28], and hence there is an $\alpha_{1}$-invariant function $\phi: G \rightarrow M(H / P)$ (see $\S 3$, and for a complete discussion see [28, Chapter 4]). Since $\Gamma \cap R$ acts ergodically on $R_{1}$ and $H$ act smoothly on $M(H / P)$ in the sense of [28] (i.e. all orbits are locally closed; see [28, Chapter 3] for a proof) we can apply the cocycle reduction lemma [28, 4.2.11]. This implies that there is an $\alpha_{1}$-invariant function $\phi: R_{1} \times(\Gamma \cap R) \rightarrow H / H_{1}$ where $H_{1}$ is the stabilizer of an element of $M(H / P)$. By a result of Moore [18] (see also [28, Chapter 3]), $H_{1}$ is an amenable algebraic group. Choose $A \subset H$ to be an amenable algebraic group of minimal dimension for which there is an $\alpha_{1}$-invariant $\phi: R_{1} \rightarrow H / A$. Since the $\Gamma \cap R$-actions on all the fibers of $q: G \rightarrow E$ are mutually isomorphic, we can find a measurable $\alpha_{1}$-invariant $\phi: G \times(\Gamma \cap R) \rightarrow H / A$.

Now let $N\left(A^{0}\right)$ be the normalizer in $H$ of the identity component of $A$, so that $N\left(A^{0}\right)$ is also an algebraic subgroup of $H$. Let $p: H / A \rightarrow H / N\left(A^{0}\right)$ be the natural map. The uniqueness argument of $[28,6.2 .8]$, using the minimality of the dimension property of $A$, shows that if $\phi_{1}, \phi_{2}$ are both $H / A$-valued $\alpha_{1}$-invariant functions on $R_{1}$, then $p \circ \phi_{1}=p \circ \phi_{2}$. It follows that if $\phi_{1}, \phi_{2}: G \rightarrow$ $H / A$ are $\alpha_{1}$-invariant, then $p \circ \phi_{1}=p \circ \phi_{2}$. Since $\Gamma$ normalizes $\Gamma \cap R$, if $\phi: G \rightarrow H / A$ is $\alpha_{1}$-invariant, then $\phi \cdot \gamma$ will be $\alpha_{1}$-invariant as well for any $\gamma \in \Gamma$. Here $\phi \cdot \gamma$ is given by $(\phi \cdot \gamma)(g)=\phi(g \gamma) \alpha(g, \gamma)^{-1}$. Thus, $p \circ \phi=p \circ(\phi \cdot \gamma)$. In other words, $p \circ \phi$ is actually $\alpha$-invariant, not just $\alpha_{1}$-invariant. We thus have an $\alpha$-invariant map $G \rightarrow H / N\left(A^{0}\right)$. Since $\alpha$ is Zariski dense in $G$, this implies $N\left(A^{0}\right)=H$, and since $A$ is amenable (and $H$ has no compact factors), we have that $A^{0}$ is trivial, i.e. $A$ is finite.

Thus, we may suppose $\alpha_{1}=\alpha \mid G \times(\Gamma \cap R)$ is equivalent to a cocycle taking all values in a finite subgroup $A \subset H$. Let $\psi: G \rightarrow H$ be measurable with $\beta(g, \gamma)=\psi(g) \alpha(g, \gamma) \psi(g \gamma)^{-1} \in A$ for all $g \in G, \gamma \in \Gamma \cap R$. We recall that $\alpha$ satisfies $\theta(g \gamma)=\theta(g) \alpha(g, \gamma)$, where $\theta: G \rightarrow X$. Thus, if we define $\lambda: G \rightarrow X$ by $\lambda(g)=\theta(g) \cdot \psi(g)^{-1}$, we have $\lambda(g) \beta(g, \gamma)=\lambda(g \gamma)$. Thus, $[\lambda(g)]=[\lambda(g \gamma)]$ in $X / A$. Since $\Gamma \cap R$ is ergodic on the fibers of $q$, and $X / A$ is a countably separated Borel space owing to the finiteness of $A[28,2.1 .21],[\lambda]$ is constant on the fibers of $q$, i.e., all $\lambda(g), g \in R_{1}$, lie in the same $A$-orbit. Hence, all $\theta(g), g \in R_{1}$, lie in the same $H$-orbit. However, by the choice of $\theta$, this means $\Gamma \cap R$ acts transitively on $R_{1}$, i.e. $\Gamma \cap R=R_{1}$. Hence $\Gamma \cap R$ is closed, and therefore discrete.

Corollary 5.3. If the $H$-action is essentially free, $\Gamma \cap R$ is central in $G$.

Proof. By Lemma 5.2, it is discrete. Since it is normalized by $\Gamma$ and $\Gamma$ is dense in $G$, it is also normal in $G$.

Corollary 5.4. To prove Theorems $\mathrm{A}$ and $\mathrm{D}$, it suffices to assume $\Gamma \cap R$ is trivial. 
Proof. Let $G^{\prime}=G / \Gamma \cap R$ and $\Gamma^{\prime}=\Gamma / \Gamma \cap R$. Then $\Gamma^{\prime} \subset G^{\prime}$ is a dense subgroup, and the action of $\Gamma^{\prime}$ on $G^{\prime}$ is stably orbit equivalent to the action of $\Gamma$ on $G$. In the case of Theorem A, if $\mathscr{F}$ has good development over $G$, and $\Gamma$ is the holonomy group in $G$, then $\mathscr{F}$ will have good development over $G^{\prime}$ and $\Gamma^{\prime}$ will be the holonomy group in $G^{\prime}$. It follows readily that if we obtain the desired results for $G^{\prime}$ and $\Gamma^{\prime}$, we have them for $G$ and $\Gamma$ as well.

\section{HOMOMORPHISMS OF $\Gamma$ INTO SIMPLE LIE GROUPS}

We continue with the notation established at the beginning of $\S 5$. We let $p: G \rightarrow G / R=L$ be projection, and let $\Lambda=p(\Gamma)$. Thus, $L$ is nontrivial (by Lemma 5.1) and $\Lambda \subset L$ is a countable dense subgroup of a connected semisimple adjoint Lie group. If $Q$ is a locally compact group and $h: \Gamma \rightarrow Q$ is a homomorphism, we let $\alpha_{h}: G \times \Gamma \rightarrow Q$ be the cocycle $\alpha_{h}(g, \gamma)=h(\gamma)$. Our main goal in this section is Theorem 6.4, which asserts that if $Q$ is a noncompact simple adjoint Lie group, then the number of homomorphisms of $\Gamma$ into $Q$ (modulo conjugacy in $Q$ ) with Zariski dense image is finite. Basic to proving this is understanding the relation between $h$ and $\alpha_{h}$.

We first need the following easy fact.

Lemma 6.1. Suppose that for $i=1,2, Q_{i}$ is a connected simple adjoint (real or complex) Lie group, and $h_{i}: \Gamma \rightarrow Q_{i}$ is a homomorphism with $h_{i}(\Gamma)$ Zariski dense in $Q_{i}$. Let $h=\left(h_{1}, h_{2}\right): \Gamma \rightarrow Q_{1} \times Q_{2}$ and let $Q$ be the algebraic hull of $h(\Gamma)$. Then either:

(i) $Q=Q_{1} \times Q_{2}$; or

(ii) $Q^{0}$ is the graph of a smooth isomorphism $\pi: Q_{1} \rightarrow Q_{2}$, and hence $\pi \circ h_{1}=h_{2}$.

Proof. Since $Q_{i}$ is connected and $h_{i}(\Gamma)$ is Zariski dense, $Q^{0}$ projects onto both $Q_{1}$ and $Q_{2}$. Write $Q^{0}=R \ltimes U$ where $R$ is reductive and $U$ is unipotent. Then the projection of $U$ into $Q_{i}$ is normal and unipotent, hence trivial. Similarly, the projection of the center $Z(R)$ onto $Q_{i}$ is central and hence trivial. Thus, $Q^{0}$ is semisimple and adjoint. Hence, there is $\widetilde{Q}_{i} \subset Q^{0}$ which is normal such that $\widetilde{Q}_{i}$ maps isomorphically onto $Q_{i}$. If $\widetilde{Q}_{1}=\widetilde{Q}_{2}$, we clearly have conclusion (ii). If not, $Q^{0} \supset \widetilde{Q}_{1} \times \widetilde{Q}_{2}$, and we clearly have assertion (i).

Theorem 6.2. Let $G, L, \Gamma$ be as above and $Q$ a connected simple adjoint (real or complex) Lie group. For $i=1,2$, let $h_{i}: \Gamma \rightarrow Q$ be a homomorphism with $h_{i}(\Gamma)$ Zariski dense in $Q$. Suppose $\alpha_{h_{1}} \sim \alpha_{h_{2}}$. Then either:

(a) there is a continuous automorphism $\pi$ of $Q$ such that $\pi \circ h_{1}=h_{2}$; or

(b) for $i=1,2, h_{i}$ extends to a continuous homomorphism $h_{i}: G \rightarrow Q$, and hence defines, in the real case, a rational surjection $\bar{h}_{i}: L \rightarrow Q$, and in the complex case a rational surjection $L_{\mathrm{C}} \rightarrow Q$. 
Proof. Let $Q_{1} \subset Q \times Q$ be given by $Q_{1}=\{(x, x) \mid x \in Q\}$, so that $Q_{1} \subset Q \times Q$ is an algebraic subgroup. Then $h_{1}$ and $h_{2}$ are conjugate by an element of $Q$ if and only if the homomorphism $h=\left(h_{2}, h_{1}\right): \Gamma \rightarrow Q \times Q$ is contained in a $Q \times Q$-conjugate of $Q_{1}$. Now assume $\alpha_{h_{1}} \sim \alpha_{h_{2}}$. Then there is a measurable map $\phi: G \rightarrow Q$ such that $\phi(g) h_{1}(\gamma) \phi(g \gamma)^{-1}=h_{2}(\gamma)$ for (almost) all $g \in G$, and all $\gamma \in \Gamma$. The group $Q \times Q$ acts on $Q$ by $z \cdot(x, y)=x^{-1} z y$. This action is transitive and the stabilizers are precisely the conjugates of $Q_{1}$ in $Q \times Q$. The cohomology equation above can be rewritten as $\phi(g \gamma)=\phi(g) \cdot\left(h_{2}(\gamma), h_{1}(\gamma)\right)$. In other words, $\phi: G \rightarrow Q$ is a $\Gamma$-map where $Q$ is considered as the homogeneous space $Q=(Q \times Q) / Q_{1}$, and $\Gamma$ acts on $Q$ via $h$. By Lemma 6.1, if $h(\Gamma)$ is not Zariski dense in $Q \times Q$, we have conclusion (a). Thus, we may assume Zariski density. Since $Q_{1}$ contains no nontrivial normal subgroup of $Q \times Q$, we can apply Corollary 4.2 and deduce that $h$ (and hence $h_{1}$ and $h_{2}$ ) extends continuously to $G$.

We shall apply this result to study $\operatorname{Hom}(\Gamma, Q)$. We first observe:

Lemma 6.3. If $Q$ is a connected simple adjoint (real or complex) Lie group, and $h: \Gamma \rightarrow Q$ is a homomorphism with $h(\Gamma)$ Zariski dense, then the following are equivalent:

(1) $h$ does not extend to $G$; and

(2) $\alpha_{h}$ is Zariski dense in $Q$.

Proof. If $\alpha_{h}$ is not Zariski dense, then there is an $\alpha_{h}$ invariant $\phi: G \rightarrow Q / Q_{1}$, where $Q_{1} \subset Q$ is proper algebraic. This simply means that $\phi$ is a $\Gamma$-map. Since $h(\Gamma)$ is Zariski dense we can apply Corollary 4.2 to deduce that $h$ extends to $G$. Conversely, if $h$ extends, we have $h(g) \alpha_{h}(g, \gamma) h(g \gamma)^{-1}=e$, so that $\alpha_{h}$ has algebraic hull $\{e\}$.

Theorem 6.4 (with notation as in the beginning of §5). For any noncompact, connected, simple, adjoint Lie group $Q$, the number of conjugacy classes of homomorphisms $\Gamma \rightarrow Q$ with Zariski dense image is finite. Furthermore, if there is such a homomorphism which does not extend to $G$, then $Q$ is a factor of $H$.

Proof. The homomorphisms $h: \Gamma \rightarrow Q$ with Zariski dense image fall into two groups: (I) those with $\alpha_{h}$ Zariski dense, and (II) those with $\alpha_{h}$ not Zariski dense. By Lemma 6.3, those in group (II) extend to continuous surjective homomorphisms $G \rightarrow Q$, and these factor to surjections $L \rightarrow Q$. Thus, there are only finitely many conjugacy classes in group (II). For those in group (I), we have from Theorem 3.12 and Corollary 3.10 that $\left\{\alpha_{h} \mid h\right.$ in group (I) $\}$ forms only finitely many equivalence classes of cocycles, and that if this set is nonempty, then $Q$ is a factor of $H$. By Theorem 6.2, this implies $\{h \mid h$ in group (I) $\}$ is finite modulo $\operatorname{Aut}(Q)$, and since $Q$ is simple, it is finite modulo conjugacy by elements of $Q$. 
7. HOMOMORPHISMS INTO TOTALLY DISCONNECTED SIMPLE GROUPS

Theorem 7.1. Let $G, \Gamma$ as above. Let $M$ be a $k$-simple connected $k$-group, where $k$ is a totally disconnected local field of characteristic 0 . Then for any homomorphism $\pi: \Gamma \rightarrow M_{k}$ with $\pi(\Gamma)$ Zariski dense we have $\overline{\pi(\Gamma)}$ is compact.

Proof. We may clearly assume $M$ is an adjoint group. As above, let $\alpha_{\pi}$ be the cocycle $\alpha_{\pi}: G \times \Gamma \rightarrow M_{k}$ given by $\alpha_{\pi}(g, \gamma)=\pi(\gamma)$.

Lemma 7.2. $\alpha_{\pi}$ is Zariski dense in $M_{k}$.

Proof. If not, there is an $\alpha_{\pi}$-invariant function $\phi: G \rightarrow M_{k} / N_{k}$ where $N_{k} \subset$ $M_{k}$ is (the $k$-points of ) a proper $k$-subgroup, i.e., $\phi$ is a $\Gamma$-map. However, this contradicts Corollary 4.3.

Continuing the proof of 7.1, we may now apply assertion (c) of Theorem 3.12 and Corollary 3.10. We deduce that $\alpha_{\pi}$ is equivalent to a cocycle taking all values in a compact subgroup of $M_{k}$. We are thus reduced to proving:

Lemma 7.3. Let $k$ be a local field of characteristic $0, M$ an (algebraically) connected $k$-simple adjoint $k$-group, and $\pi: \Gamma \rightarrow M_{k}$ a homomorphism with $\pi(\Gamma)$ Zariski dense. Suppose $\alpha_{\pi}$ is Zariski dense and that $\alpha_{\pi}$ is equivalent to a cocycle taking all values in a compact subgroup of $M_{k}$. Then $\overline{\pi(\Gamma)}$ is compact.

Proof. Let $Q \subset M$ be a minimal parabolic $k$-subgroup. Thus, $M_{k} / Q_{k}$ is compact. Let $K \subset M_{k}$ be a compact subgroup such that there is a measurable $\phi: G \rightarrow M_{k}$ for which $\phi(g) \alpha_{\pi}(g, \gamma) \phi(g \gamma)^{-1} \in K$. Let $M\left(M_{k} / Q_{k}\right)$ be the space of probability measures on $M_{k} / Q_{k}$ with the weak- ${ }^{*}$ topology. Since $K$ is compact, there is a $K$-invariant $\mu \in M\left(M_{k} / Q_{k}\right)$. Let $\lambda: G \rightarrow M\left(M_{k} / Q_{k}\right)$ be defined by $\lambda(g)=\mu \cdot \phi(g)$, where we write the action of $M_{k}$ on $M\left(M_{k} / Q_{k}\right)$ on the right. Then $K$-invariance of $\mu$ implies that $\lambda$ is $\alpha_{\pi}$-invariant. Equivalently, $\lambda$ is a $\Gamma$-invariant element of the function space $F\left(G, M\left(M_{k} / Q_{k}\right)\right)$, where, as usual, this is the space of measurable functions, two being identified if they agree almost everywhere. The $\Gamma$ action is given by right translation on $G$ and the action on $M\left(M_{k} / Q_{k}\right)$ given by $\pi$. The action of $G$ on $F\left(G, M\left(M_{k} / Q_{k}\right)\right)$ given by $(g \cdot f)(a)=f\left(g^{-1} a\right)$ commutes with the $\Gamma$-action. In particular, if we let $A=F\left(G, M\left(M_{k} / Q_{k}\right)\right)^{\Gamma}$ be the space of $\Gamma$-invariants, then $A$ is a compact convex set (with the weak- ${ }^{*}$ topology as in [25]; see [25] for a full discussion) on which $G$ acts linearly and continuously. Let $p: G \rightarrow L$ be the projection. Let $P \subset L$ be the product of the compact factors of $L$ together with a minimal parabolic subgroup for each noncompact factor of $L$. (If $L$ is compact, $P=L$. This is degenerate for the remainder of the argument, but the argument still applies.) Since $P$ is amenable and $\operatorname{ker} p=R$ is solvable, $P^{*}=p^{-1}(P)$ is also amenable. Hence, there is a $P^{*}$-invariant element in $A$. Therefore, if we let $\widetilde{\alpha}_{\pi}$ be the cocycle $\widetilde{\alpha}_{\pi}: P \backslash L \times \Gamma \rightarrow M_{k}$ given by $\widetilde{\alpha}_{\pi}(x, \gamma)=\pi(\gamma)$, such a $P^{*}$-invariant element of $A$ will define a $\widetilde{\alpha}_{\pi}$-invariant 
function $\psi: P \backslash L \rightarrow M\left(M_{k} / Q_{k}\right)$. The $M_{k}$ action on $M\left(M_{k} / Q_{k}\right)$ has locally closed orbits and the stabilizers are either compact or contained in the $k$-points of a proper algebraic $k$-subgroup of $M_{k}$ [28, Chapter 3]. Since $\Gamma$ is dense in $G$ (and hence in $L$ ), it acts ergodically on $P \backslash L$. Therefore, as in [28, p. 93], we deduce that either:

(a) there is a measurable $\Gamma$-map $\psi: P \backslash L \rightarrow M_{k} / N_{k}$ where $N$ is a proper $k$-subgroup; or

(b) there is a measurable $\Gamma$-map $\psi: P \backslash L \rightarrow M_{k} / B$ where $B \subset M_{k}$ is a compact subgroup.

Case (a) is impossible since $\alpha_{\pi}$ is Zariski dense in $M_{k}$. In case (b), we observe that $L$ has a conull orbit in $P \backslash L \times P \backslash L$, and hence $\Gamma$ acts ergodically on $P \backslash L \times P \backslash L$. From [28, 5.9.1], we see that (b) implies that $\overline{\pi(\Gamma)}$ is compact.

\section{HOMOMORPHISMS OF $\Gamma$ INTO COMPLEX SIMPLE GROUPS}

The arguments of $\S \S 6$ and 7 now allow us to prove the following result about homomorphisms into complex groups.

Theorem 8.1. Let $\Gamma, G$ as above. Let $Q$ be a connected simple adjoint algebraic group (over $\mathbf{C}$ ). Then the number of conjugacy classes of homomorphisms $h: \Gamma \rightarrow Q$ such that (i) $h(\Gamma)$ is Zariski dense in $Q$, and (ii) $\overline{h(\Gamma)}$ is not compact, is finite.

Proof. As in the proof of Theorem 6.4, we consider the two groups of homomorphisms $h: \Gamma \rightarrow Q$ with $h(\Gamma)$ Zariski dense: (I), those $h$ with $\alpha_{h}$ Zariski dense in $Q$; and (II), those with $\alpha_{h}$ not Zariski dense. By Lemma 6.3, those in group (II) extend to continuous homomorphisms $G \rightarrow Q$, and to continuous surjections $L_{\mathrm{C}} \rightarrow Q$. Thus, there are only finitely many conjugacy classes in group (II). For those in group (I) with the additional property that $\overline{h(\Gamma)}$ is not compact, we have from Lemma 7.3 that $\alpha_{h}$ is not equivalent to a cocycle into a compact subgroup of $Q$. Therefore, by Theorem 3.12(b), those $\left\{\alpha_{h}\right\}$ in group (I) with $\overline{h(\Gamma)}$ not compact form only finitely many equivalence classes of cocycles. By Theorem 6.2 , we deduce that there are only finitely many conjugacy classes of such homomorphisms.

\section{ARITHMETICITY IN THE SEMISIMPLE COMPONENT, AND THE PROOF OF THEOREM B}

In this section we prove that $\Lambda$, the image of $\Gamma$ in $L$, is a dense arithmetic subgroup of $L$, under the additional assumption that the $H$-action on $X$ is essentially free. Along the way, without this additional assumption, we shall prove Theorem B. The first step in proving arithmeticity is standard, given the homomorphism results of $\S \S 6-8$. We continue our notation from preceding sections. (We do not yet assume essential freeness of the $H$-action.) 
Lemma 9.1. There is a number field $k, Q \subset k \subset \mathbf{R},[k: \mathbf{Q}]<\infty$, with ring of integers $\mathcal{O}$, a semisimple adjoint $k$-group $W$, and an isomorphism of Lie groups $L \cong W_{\mathbf{R}}^{0}$ such that for some subgroup $\Lambda^{\prime} \subset \Lambda$ of finite index, we have $\Lambda^{\prime} \subset W_{\mathscr{Q}}$.

Proof. We follow the general outline of [28, Chapter 6]. We first claim $\operatorname{Tr}\left(\operatorname{Ad}_{L}(\gamma)\right) \in \overline{\mathbf{Q}}$ for all $\gamma \in \Lambda$. For this, it clearly suffices to see $\operatorname{Tr}\left(\operatorname{Ad}_{L_{1}}\left(p_{1}(\gamma)\right)\right)$ $\in \overline{\mathbf{Q}}$ for all $\gamma \in \Lambda$, and any simple factor $p_{1}: L \rightarrow L_{1}$ of $L$. We can write $L_{1}=M_{\mathbf{R}}^{0}$ where $M \subset \mathrm{GL}(n, \mathbf{C})$ is an $\mathbf{R}$-simple adjoint $\mathbf{Q}$-group. For any $\sigma \in \mathrm{Gal}(\mathbf{C} / \mathbf{Q})$ we have a (discontinuous in general) automorphism of $\mathrm{GL}(n, \mathbf{C})$, and since $M$ is a $\mathbf{Q}$-group, we have an automorphism of $M$ and $\operatorname{Ad}_{M}(M)$. We denote these automorphisms by $\sigma$ as well. Let $h_{\sigma}: \Gamma \rightarrow M$ be the composition $\Gamma \rightarrow \Lambda \hookrightarrow L \rightarrow L_{1} \rightarrow M \stackrel{\sigma}{\longrightarrow} M$. Then $h_{\sigma}(\Gamma)$ is Zariski dense in $M$. Suppose $\operatorname{Tr}\left(\operatorname{Ad}_{L_{1}}\left(p_{1}(\gamma)\right)\right)$ is transcendental for some $\gamma \in \Lambda$. Then $\left\{\sigma\left(\operatorname{Tr}\left(\operatorname{Ad}_{L_{1}}\left(p_{1}(\gamma)\right)\right)\right) \mid \sigma \in \operatorname{Gal}(\mathbf{C} / \mathbf{Q})\right\}$ is dense in $\mathbf{C}$. This set is of course identical to $\left\{\operatorname{Tr}\left(\operatorname{Ad}_{M}\left(h_{\sigma}(\gamma)\right)\right) \mid \sigma \in \operatorname{Gal}(\mathbf{C} / \mathbf{Q})\right\}$ (where we lift $\gamma$ back to an element of $\Gamma$ ). This implies that for infinitely many such $\sigma, h_{\sigma}(\Gamma)$ is not contained in a compact subgroup of $M$, and that among these, there are infinitely many nonconjugate $h_{\sigma}$. This contradicts Theorem 8.1.

Given that these traces are all algebraic, the lemma of Vinberg (see $[28,6.1 .7])$ implies that we can realize $L$ as $L=W_{\mathbf{R}}^{0}$ where $W$ is a $\overline{\mathbf{Q}} \cap \mathbf{R}$-group, and $\Lambda \subset W_{\overline{\mathbf{Q}} \cap \mathbf{R}}$.

Since $\Lambda$ is finitely generated, we have $\Lambda \subset W_{k}$ for some $k \subset \mathbf{R}$ with [ $k: \mathbf{Q}]<\infty$, and since $\Lambda$ is Zariski dense, $W$ is defined over $k$. Once again using the fact that $\Lambda$ is finitely generated, we can find finitely many (finite) primes $\mathscr{P}_{i}$ of $\mathscr{O}$ such that, denoting by $k_{i}$ (resp., $\mathscr{O}_{i}$ ) the completion of $k$ (resp., $\mathscr{O}$ ) at $\mathscr{P}_{i}$, we have that the map $\Lambda / \Lambda \cap W_{\mathscr{O}} \rightarrow \prod_{i} W_{k_{i}} / W_{\mathscr{O}_{i}}$ is injective. Since $k_{i}$ is totally disconnected, $W_{\mathscr{O}_{i}}$ is a compact open subgroup, and since the map $\Lambda \rightarrow W_{k_{i}}$ has Zariski dense image, it follows from Theorem 7.1 that for each $i$ the image of $\Lambda$ in $W_{k_{i}} / W_{\mathscr{O}_{i}}$ is finite. Therefore $\Lambda^{\prime}=\Lambda \cap W_{\mathscr{O}}$ is of finite index in $\Lambda$. This proves the lemma.

We now consider the Q-group $R_{k / \mathbf{Q}}(W)$ obtained from $W$ by restriction of scalars [28, Chapter 6]. We let $J=R_{k / \mathbf{Q}}(W)_{\mathbf{R}}^{0}$. Then $J$ is a connected semisimple adjoint Lie group. The group $L$ is a factor of $J$ and we have a projection $r: J \rightarrow L$ such that $r\left(J_{\mathbf{Q}}\right)=W_{k} \cap L$, and $r\left(J_{\mathbf{Z}}\right)=W_{\mathscr{Q}} \cap L$. There is also an isomorphism $\alpha: W_{k} \rightarrow R_{k / \mathbf{Q}}(W)_{\mathbf{Q}}$ such that $\alpha\left(W_{\mathscr{O}}\right)=R_{k / \mathbf{Q}}(W)_{\mathbf{z}}$ (see [28] for details).

Let $M^{*}$ be the algebraic hull of $\alpha\left(\Lambda^{\prime}\right)$ in $R_{k / Q}(W)$. Since the projection of $\alpha\left(\Lambda^{\prime}\right)$ onto each simple factor of $J$ is Zariski dense, the proof of Lemma 6.1 implies that $M^{*}$ is semisimple and adjoint, and since $\alpha\left(\Lambda^{\prime}\right) \subset J_{\mathbf{Q}}, M^{*}$ is a Q-group. Let $M=\left(M_{\mathrm{R}}^{*}\right)^{0}$. By passing to a normal subgroup $\Lambda^{\prime \prime} \subset \Lambda$ of finite index, we can assume $\alpha\left(\Lambda^{\prime \prime}\right) \subset M_{\mathbf{Z}}^{*} \cap M$. Since $r\left(\alpha\left(\Lambda^{\prime \prime}\right)\right)$ is Zariski dense in 
$L$, we have that $M$ projects onto $L$, so we can write $M=L \times L^{\prime} \times K$ where $L^{\prime}$ is the product of the remaining noncompact simple factors of $M$, and $K$ is the product of the remaining compact simple factors. Clearly the projection of $\alpha\left(\Lambda^{\prime \prime}\right)$ into $L \times L^{\prime}$ is discrete. We choose a normal subgroup $L^{\prime \prime} \subset L^{\prime}$ such that the projection of $\alpha\left(\Lambda^{\prime \prime}\right)$ of $L \times L^{\prime \prime}$ is discrete, but the projection into $L \times L^{\prime \prime \prime}$ is not discrete for any proper normal subgroup $L^{\prime \prime \prime} \subset L^{\prime \prime}$. We remark that since $\Lambda$ (and hence $\Lambda^{\prime \prime}$ ) is dense in $L, L^{\prime \prime}$ is not trivial. Recall the map $p: G \rightarrow L$, and let $\Gamma^{\prime \prime}=p^{1}\left(\Lambda^{\prime \prime}\right)$, so that $\Gamma^{\prime \prime} \subset \Gamma$ is normal and of finite index. We wish to apply our earlier results to the action of $\Gamma^{\prime \prime}$ on $G$. Therefore, we need:

Lemma 9.2. The $\Gamma^{\prime \prime}$ action on $G$ is stably orbit equivalent to an ergodic action of $H$ preserving a finite invariant measure.

Proof. Since $G$ is connected, $\Gamma^{\prime \prime}$ is clearly dense in $G$. Let $c$ be the cocycle of the $\Gamma$-action on $G$ defined by the homomorphism $\lambda: \Gamma \rightarrow \Gamma / \Gamma^{\prime \prime}$, i.e. $c(g, \gamma)=$ $\lambda(\gamma)$. By Corollary 3.10 and the discussion preceding it, $c$ corresponds to an orbital cocycle $\tilde{c}: X \times H \rightarrow \Gamma / \Gamma^{\prime \prime}$. It is not hard to verify that the action of $H$ on $X \times \Gamma / \Gamma^{\prime \prime}$ given by $(x,[\gamma]) \cdot h=(x h,[\gamma] \widetilde{c}(x, h))$ is then stably orbit equivalent to the action of $\Gamma$ on $G \times \Gamma / \Gamma^{\prime \prime}$. This latter action is in turn stably equivalent to the action of $\Gamma^{\prime \prime}$ on $G$. Finally, we remark that the action of $H$ on $X \times \Gamma / \Gamma^{\prime \prime}$ is ergodic as ergodicity is an invariant of stable orbit equivalence.

Proof of Theorem B. Let $Q$ be an R-simple factor of $L^{\prime \prime}$. Then $Q$ must project injectively into some factor $W^{\sigma}$ of $R_{k / \mathbf{Q}}(W)=\prod_{\sigma} W^{\sigma}$ where $\sigma$ runs through the distinct embeddings of $k$ in $\mathbf{C}$. Thus, $\operatorname{dim}_{\mathbf{R}} Q \leq \operatorname{dim}_{\mathbf{R}} W^{\sigma}=$ $2 \operatorname{dim}_{\mathbf{R}} L$. Now consider the homomorphism $\pi_{Q}: \Gamma^{\prime \prime} \rightarrow Q$ which is the composition of $p \circ \alpha$ with projection onto $Q$. This has Zariski dense image. Furthermore, $\pi_{Q}$ does not extend to a continuous homomorphism of $G$, for otherwise such a homomorphism would define a rational homomorphism $\bar{\pi}_{Q}: L \rightarrow Q$, and projection of $\alpha\left(\Lambda^{\prime \prime}\right)$ onto $L \times Q$ would be contained in the graph of $\bar{\pi}_{Q}$. This would contract Zariski density of $\alpha\left(\Lambda^{\prime \prime}\right)$ in $M$. It now follows from Lemma 6.3 that the cocycle $\alpha_{\pi_{Q}}: G \times \Gamma^{\prime \prime} \rightarrow Q$ is Zariski dense in $Q$. By Corollary 3.10, Lemma 9.2, and Theorem 3.12, we deduce that there is a rational surjection $H \rightarrow Q$. In particular, $\operatorname{dim} Q \geq d(H)=\min \left\{\operatorname{dim} H^{\prime} \mid H^{\prime}\right.$ is a simple factor of $H\}$. Therefore, $\operatorname{dim} L \geq d(H) / 2$, completing the proof of Theorem B.

We now return to the situation in Theorems A and D. We shall henceforth assume, by virtue of Corollary 5.4, that $\Gamma \cap R$ is trivial. Otherwise, we retain all the notation of this section.

Let $q: M \rightarrow L \times L^{\prime \prime}$ be projection. We can write the homomorphism $q \circ \alpha: \Lambda^{\prime \prime} \rightarrow L \times L^{\prime \prime}$ as $(q \circ \alpha)(\gamma)=\left(\gamma, \alpha^{\prime}(\gamma)\right)$ where $\alpha^{\prime}: \Lambda^{\prime \prime} \rightarrow L^{\prime \prime}$ is a homomorphism. Define $\beta: \Gamma^{\prime \prime} \rightarrow G \times L^{\prime \prime}$ by $\beta(\gamma)=\left(\gamma, \alpha^{\prime}(p(\gamma))\right)$. Then $\beta$ is an injective homomorphism and the projection of $\beta\left(\Gamma^{\prime \prime}\right)$ onto $L \times L^{\prime \prime}$ is simply $q\left(\alpha\left(\Lambda^{\prime \prime}\right)\right)$. 
In particular, this projection is discrete and since $\beta\left(\Gamma^{\prime \prime}\right) \cap \operatorname{ker}\left(G \times L^{\prime \prime} \rightarrow L \times L^{\prime \prime}\right)$ is $\left(\Gamma^{\prime \prime} \cap R\right) \times\{e\}$ and hence trivial, we have that $\beta\left(\Gamma^{\prime \prime}\right) \subset G \times L^{\prime \prime}$ is discrete as well.

Lemma 9.3. The $L^{\prime \prime}$-action on $\left(G \times L^{\prime \prime}\right) / \beta\left(\Gamma^{\prime \prime}\right)$ is stably orbit equivalent to an ergodic action of $H$ preserving a finite invariant measure. Furthermore, this action of $L^{\prime \prime}$ is Zariski dense in $L^{\prime \prime}$.

Proof. For the first assertion, we begin by observing that the $L^{\prime \prime}$ action on $\left(G \times L^{\prime \prime}\right) / \beta\left(\Gamma^{\prime \prime}\right)$ is stably orbit equivalent to the $\beta\left(\Gamma^{\prime \prime}\right)$ action on $\left(G \times L^{\prime \prime}\right) / L^{\prime \prime}$. The projection of $G \times L^{\prime \prime}$ onto $G$ takes $\beta\left(\Gamma^{\prime \prime}\right)$ injectively onto $\Gamma^{\prime \prime}$, so the action in question is stably orbit equivalent to the action of $\Gamma^{\prime \prime}$ on $G$. Thus, the first assertion of Lemma 9.3 follows from Lemma 9.2.

We now turn to the second assertion of Lemma 9.3. Suppose there is a measurable $L^{\prime \prime}$-map $\phi:\left(G \times L^{\prime \prime}\right) / \beta\left(\Gamma^{\prime \prime}\right) \rightarrow L^{\prime \prime} / T$ where $T \subset L^{\prime \prime}$ is a proper algebraic subgroup. Choose $T \subset L^{\prime \prime}$ to be of minimal dimension among those algebraic subgroups for which there exists such a map. By the uniqueness argument of $[28,6.2 .8]$ (cf. the proof of Lemma 5.2), if $\phi_{1}, \phi_{2}$ are two such maps, then $\omega \circ \phi_{1}=\omega \circ \phi_{2}$ where $\omega: L^{\prime \prime} / T \rightarrow L^{\prime \prime} / N_{L^{\prime \prime}}\left(T^{0}\right)$ is projection. Since $G$ commutes with $L^{\prime \prime}, g \circ \phi$ is also an $L^{\prime \prime}$-map, and hence $\omega \circ(g \cdot \phi)=\omega \circ \phi$. Therefore, $\omega \circ \phi$ is a $G$-map as well as an $L^{\prime \prime}$-map. We deduce that $\beta\left(\Gamma^{\prime \prime}\right) \subset G \times N_{L^{\prime \prime}}\left(T^{0}\right)$. Since the projection of $\beta\left(\Gamma^{\prime \prime}\right)$ into $L^{\prime \prime}$ is Zariski dense, it follows that $T^{0} \subset L^{\prime \prime}$ is normal. Write $L^{\prime \prime}=T^{0} \times T_{1}$ where $T_{1} \subset L^{\prime \prime}$ is also normal. Then $T_{1}$ acts with closed orbits and finite stabilizers on $L^{\prime \prime} / T$. (Recall $T^{0} \subset T$ is of finite index.) It follows that (perhaps by passing to a conull set) the action of $T_{1}$ on $\left(G \times L^{\prime \prime}\right) / \beta\left(\Gamma^{\prime \prime}\right)$ is smooth in the sense of [28, Chapter 2], and hence that the $\beta\left(\Gamma^{\prime \prime}\right)$ action on $\left(G \times L^{\prime \prime}\right) / T_{1}$ is smooth, i.e., the $\beta\left(\Gamma^{\prime \prime}\right)$ action on $G \times T^{0}$ is smooth. Since $\beta\left(\Gamma^{\prime \prime}\right)$ is a countable subgroup of $G \times T^{0}$, this implies that the image of $\beta\left(\Gamma^{\prime \prime}\right)$ in $G \times T^{0}$ is discrete. Since $R \subset G$ is a solvable normal subgroup, this implies that the image of $\beta\left(\Gamma^{\prime \prime}\right)$ in $G / R \times T^{0}=L \times T^{0}$ is also discrete. (Namely, discreteness of $\beta\left(\Gamma^{\prime \prime}\right)$ implies that the action of $\beta\left(\Gamma^{\prime \prime}\right)$ on $G \times T^{0}$ is amenable [28]. Since $R$ is solvable normal, the action of $\beta\left(\Gamma^{\prime \prime}\right)$ on $(G / R) \times T_{0}$ is also amenable; cf. [31]. The main result of [31] implies that the identity component of the closure of the image of $\beta\left(\Gamma^{\prime \prime}\right)$ (i.e. the image of $\left.\alpha\left(\Lambda^{\prime \prime}\right)\right)$ in $L \times T^{0}$ is solvable. Call this connected solvable group $S$. It is of course normalized by the image of $\alpha\left(\Lambda^{\prime \prime}\right)$. However, $\alpha\left(\Lambda^{\prime \prime}\right)$ is Zariski dense in $M$, which implies that $S$ is normal in $L \times T^{0}$. It must therefore be trivial, so we deduce that the image of $\alpha\left(\Lambda^{\prime \prime}\right)$ in $L \times T^{0}$ is discrete; cf. Auslander's theorem [19].) However, by the choice of $L^{\prime \prime}$, we have $T^{0}=L^{\prime \prime}$, so $T=L^{\prime \prime}$, verifying Zariski density, and completing the proof of the lemma.

Theorem 9.4. $\Lambda \subset L$ is a dense arithmetic subgroup. Furthermore, $\Lambda^{\prime \prime}$ is isomorphic to a lattice in $L \times H$. 
Proof. We first claim that the discrete subgroup $q\left(\alpha\left(\Lambda^{\prime \prime}\right)\right) \subset L \times L^{\prime \prime}$ is actually a lattice. Since we have a surjective $G \times L^{\prime \prime}$-map

$$
\left(G \times L^{\prime \prime}\right) / \beta\left(\Gamma^{\prime \prime}\right) \rightarrow\left(L \times L^{\prime \prime}\right) / q\left(\alpha\left(\Lambda^{\prime \prime}\right)\right),
$$

it suffices to see that $\beta\left(\Gamma^{\prime \prime}\right)$ is a lattice in $G \times L^{\prime \prime}$. We shall need the following fact.

Lemma 9.5. Let $A, B$ be Lie groups and $D \subset A \times B$ a discrete subgroup. Suppose $B$ acts ergodically on $(A \times B) / D$ with respect to the smooth measure class. If there is a finite $B$-invariant measure $\mu$ of smooth class, then $D$ is a lattice in $A \times B$.

Proof. Since $\mu$ is a finite $B$-invariant ergodic measure, any other $B$-invariant measure in the same measure class with the same total mass must be equal to $\mu$. (Namely, for any such measure $\nu$, let $f=d \nu / d \mu$. This is $B$-invariant, hence constant.) However, for $a \in A, a_{*} \mu$ will be such a measure, and hence $\mu$ is $A$-invariant as well.

Returning to the proof of Theorem 9.4, to see that $\beta\left(\Gamma^{\prime \prime}\right)$ is a lattice, by Lemma 9.5 it suffices to prove that there is a finite $L^{\prime \prime}$-invariant measure of smooth class on $\left(G \times L^{\prime \prime}\right) / \beta\left(\Gamma^{\prime \prime}\right)$. However, this follows from Lemma 9.3 and Corollary 3.13. This also shows that $L^{\prime \prime}$ is isomorphic to $H$. To complete the proof of 9.4, it suffices to show that $L^{\prime}=L^{\prime \prime}$. For then $\operatorname{ker}(q)$ is compact and hence $\alpha\left(\Lambda^{\prime \prime}\right)$ is a lattice in $M$. Since we already know $\alpha\left(\Lambda^{\prime \prime}\right) \subset M_{\mathbf{Z}}^{*} \cap M$, we would then have an inclusion of lattices, and so $\alpha\left(\Lambda^{\prime \prime}\right)$ would be of finite index in $M_{\mathbf{Z}}^{*} \cap M$, verifying arithmeticity. If $L^{\prime} \neq L^{\prime \prime}$, then there is a noncompact simple normal subgroup $Y$ of $L^{\prime}$ which is not contained in $L^{\prime \prime}$. We recall that by construction $q \mid \alpha\left(\Lambda^{\prime \prime}\right)$ is injective and the projection of $\alpha\left(\Lambda^{\prime \prime}\right)$ into $Y$ is Zariski dense. Since $q\left(\alpha\left(\Lambda^{\prime \prime}\right)\right)$ is a lattice in $L \times H$ projecting densely to $L$, and all simple factors of $H$ have R-rank at least 2, we can apply Margulis' superrigidity theorem $[15,28]$ to deduce that there is a rational homomorphism $L \rightarrow Y$ extending the map of $q\left(\alpha\left(\Lambda^{\prime \prime}\right)\right)$ given by projecting $\alpha\left(\Lambda^{\prime \prime}\right)$ into $Y$. Then the projection of $\alpha\left(\Lambda^{\prime \prime}\right)$ into $L \times Y$ is contained in the graph of this homomorphism, contradicting Zariski density of $\alpha\left(\Lambda^{\prime \prime}\right)$ in $M$.

\section{COMPLETION OF THE PROOFS OF THEOREMS A AND D}

To prove Theorems $\mathrm{A}$ and $\mathrm{D}$, it remains only to show that $R$ is finite and central. (We are still under the assumption that $\Gamma \cap R$ is trivial, given by Corollary 5.4.) By Theorem 9.4, identifying $\Gamma$ with $\Lambda \subset L$, we can assume that $\Gamma=\rho(D)$ where $D \subset L \times H$ is a lattice, $\rho: L \times H \rightarrow L$ is projection, and $\rho \mid D$ is injective. Consider the map $p: G \rightarrow L$ of ergodic $\Gamma$-spaces. This is a principal $R$-bundle on which $\Gamma$ acts by principal bundle automorphisms. Hence, if we measurably trivialize the bundle, the $\Gamma$-action on $G$ will be given by a cocycle $\alpha: L \times \Gamma \rightarrow R$, i.e., we have measurably that $G=L \times R$ and $\Gamma$ 
acts by $(l, r) \cdot \gamma=(l \gamma, r \alpha(l, r))$. The $\Gamma$ action on $L$ is the same as the $D$-action on $L \cong(L \times H) / H$, which is stably orbit equivalent to the action of $H$ on $(L \times H) / D$. The latter is a finite measure preserving action of a Kazhdan group. By Corollary 3.10 and Theorem 3.14, the cocycle $\alpha$ is equivalent to a cocycle $\beta$ taking all values in a compact subgroup $R_{1} \subset R$. Thus, the $\Gamma$-action on $G$ is measurably equivalent to the action on $L \times R$ given by $(l, r) \cdot \gamma=(l \gamma, r \beta(l, \gamma))$. Since this action is ergodic (because $\Gamma$ is dense in $G$ ), it follows that $R_{1}=R$, i.e. $R$ is compact. Since $R$ is solvable, we deduce that $R^{0}$ is abelian. Then the map $R \rightarrow G /[G, G]$ has finite kernel. Since $\Gamma$ is isomorphic to a lattice in $L \times H$, projecting densely onto all factors of $\mathbf{R}$-rank less than 2 , the image of $\Gamma$ in the abelian group $G /[G, G]$ is finite [16], and since $\Gamma$ is dense in $G, G /[G, G]$ is finite. Thus, $R$ is finite, hence central, and this proves the theorems.

\section{Foliations BY QUATERNIONIC HYPERBoliC SPACE}

In this section we show how one can obtain results similar to Theorem B for foliations by certain rank one locally symmetric spaces, namely those for which the isometry group of the universal cover is a Kazhdan group.

Theorem 11.1. Let $\mathscr{F}$ be a Riemannian foliation of a compact manifold $M$. Assume there is a metric on $M$ for which the leaves are locally isomorphic to either a quaternionic hyperbolic space (of dimension at least 8) or to the Cayley hyperbolic plane. If $\operatorname{codim} \mathscr{F} \leq 3$, then there is a finite covering $\widetilde{M}$ of $M$ such that $\widetilde{F}$ is the foliation defined by a fiber bundle projection. If $\mathscr{F}$ is a Lie foliation with $\operatorname{codim} \mathscr{F} \leq 6$, the same conclusion is true.

The isometry group of the symmetric spaces in question are Kazhdan [14, 28]. Therefore, the discussion above for the higher rank case shows that it is sufficient to prove the following result about stable orbit equivalence.

Theorem 11.2. Let $G$ be a (nontrivial) connected Lie group with $\operatorname{dim} G \leq 6$, and $\Gamma \subset G$ a finitely generated dense subgroup. Suppose $H$ is a Kazhdan group and $(X, \mu)$ an ergodic $H$-space with finite invariant measure. Then the $\Gamma$-action on $G$ is not stably orbit equivalent to the $H$-action on $X$.

Example 11.3. Before proceeding with the proof, we observe that we may have a connected Lie group $G$ and a fintiely generated dense subgroup $\Gamma \subset G$ such that neither $G$ nor $\Gamma$ is Kazhdan, but the $\Gamma$-action on $G$ is stably orbit equivalent to a finite measure preserving action of a Kazhdan group. Namely, if $\Gamma \subset G \times G^{\prime}$ is an irreducible lattice where $G^{\prime}$ is Kazhdan but $G$ is not, then the $\Gamma$-action on $G$ is stably orbit equivalent to the $G^{\prime}$-action on $\left(G \times G^{\prime}\right) / \Gamma$. One can easily have examples with $G=\mathrm{SO}(1, n)$ and $G^{\prime}=\operatorname{SO}(2, n-1)$ for $n \geq 4$.

For the proof of Theorem 11.2, we will need the following result about cocycles. 
Theorem 11.4 [29]. Let $H$ be a Kazhdan group and $(X, \mu)$ an ergodic $H$-space with a finite invariant measure. Let $k$ be a local field, $\operatorname{char}(k)=0$, and $\alpha: X \times H \rightarrow \operatorname{PSL}(2, k)$. Then $\alpha$ is equivalent to a cocycle taking all values in a compact subgroup of $\operatorname{PSL}(2, k)$.

For the proof of 11.4 , see [29].

Proof of Theorem 11.2. The proof follows the main lines of the proof of Theorem B using Theorem 11.4 in place of Theorem 3.12. We shall therefore just sketch the argument. Let $R \subset G$ be as in the proofs of Theorems A, B and D. By the remark following Lemma 5.1, we can assume $G \neq R$, so $L^{\prime}=G / R$ is nontrivial. Since $\operatorname{dim} L^{\prime} \leq 6$, there is a simple factor $L$ of $L^{\prime}$ which is a subgroup of $\operatorname{PSL}(2, \mathrm{C})$. For ease of notation, we let $\operatorname{PSL}(2, \mathrm{C})=M$; Ad will denote $\operatorname{Ad}_{M}$. Let $\Lambda$ be the (dense) image of $\Gamma$ in $L$. For each $\sigma \in \operatorname{Gal}(\mathbf{C} / \mathbf{Q})$ we obtain an automorphism (usually discontinuous) of $M$, which we still denote by $\sigma$, and hence a homomorphism $h_{\sigma}: \Gamma \rightarrow M$. This image is Zariski dense in $M$ (where $M$ is viewed as an algebraic group over $\mathbf{C}$, not as a real algebraic group). Let $\alpha_{\sigma}: G \times \Gamma \rightarrow M$ be the cocycle $\alpha_{\sigma}(g, \gamma)=h_{\sigma}(\gamma)$. Then by Lemma 6.3, either $\alpha_{\sigma}$ is Zariski dense in $M$ or $h_{\sigma}$ extends to a continuous homomorphism $G \rightarrow M$, which must factor to a homomorphism $L^{\prime} \rightarrow M$. In case $\alpha_{\sigma}$ is Zariski dense, we have from Theorem 11.4 and Lemma 7.3 that $h_{\sigma}(\Gamma)$ has compact closure. We deduce, as in the proof of Lemma 9.1, that $\operatorname{Tr}(\operatorname{Ad}(\gamma)) \in \overline{\mathbf{Q}}$ for all $\gamma \in \Lambda$. Then, as in 9.1 (see also [1, p. 135]) we may assume $\operatorname{Ad}(\Lambda) \subset \operatorname{Ad}(M)_{k}$ where $k \subset \mathbf{R}$ is a finite Galois extension of $\mathbf{Q}$. By Theorem 11.4 and Lemma 7.3, we again deduce, as in the proof of Lemma 9.1, that $\operatorname{Ad}\left(\Lambda^{\prime}\right) \subset \operatorname{Ad}(M)_{\mathscr{O}}$ for some subgroup $\Lambda^{\prime} \subset \Lambda$ of finite index, where $\mathscr{O}$ is the ring of integers in $k$. Let $\Gamma^{\prime}$ be the pull-back of $\Lambda^{\prime}$ to $\Gamma$, so $\Gamma / \Gamma^{\prime}$ is also finite. Let $\alpha: \operatorname{Ad}(M)_{\mathscr{O}} \rightarrow R_{k / \mathbf{Q}}(\operatorname{Ad}(M))_{\mathbf{Z}}$ be the standard map (see [28]). Let $N$ be the algebraic hull of $\alpha\left(\Lambda^{\prime}\right)$. Then $N$ is semisimple, projects onto $\operatorname{Ad}(M)$, and we can write $N \cong \operatorname{Ad}(M) \times M^{\prime}$. Since $\alpha\left(\Lambda^{\prime}\right)$ is discrete, and $\operatorname{Ad}\left(\Lambda^{\prime}\right)$ is not discrete, the projection of $\alpha\left(\Lambda^{\prime}\right)$ onto $M^{\prime}$ cannot be precompact. Thus, there is some R-simple factor $M^{\prime \prime}$ of $M^{\prime}$ in which the projection of $\alpha\left(\Lambda^{\prime}\right)$ is not precompact. There is some $\sigma \in \operatorname{Gal}(k / \mathbf{Q})$ so that the projection of $M^{\prime \prime} \subset R_{k / Q}(\operatorname{Ad} M)$ onto $\operatorname{Ad}(M)^{\sigma}$ is an isomorphism of Lie groups. By Theorem 11.4 and Lemma 7.3, we deduce that for such a $\sigma$, the cocycle $\alpha_{\sigma}: G \times \Gamma^{\prime} \rightarrow M^{\sigma}$ is not Zariski dense. Therefore, by Lemma $6.3, h_{\sigma}$ extends to a smooth homomorphism $G \rightarrow M^{\sigma}$, hence defines a rational homomorphism $L \rightarrow M^{\prime \prime}$. This contradicts the Zariski density of $\alpha\left(\Lambda^{\prime}\right)$ in $M$, and completes the proof.

\section{REFERENCES}

1. H. Bass, Finitely generated subgroups of $\mathrm{GL}(2)$, in the Smith Conjecture (J. Morgan and H. Bass, eds.), Academic Press, New York, 1984.

2. R. A. Blumenthal, Transversally homogeneous foliations, Ann. Inst. Fourier (Grenoble) 29 (1979), 143-158. 
3. A. Borel, Density properties for certain subgroups of semisimple Lie groups without compact factors, Ann. of Math. (2) 72 (1960), 179-188.

4. Y. Carriere, Feuilletages Riemanniens a croissance polynomiale, preprint, Lille, 1986.

5. Y. Carriere and E. Ghys, Relations d'equivalence moyenables sur les groupes de Lie, C. R. Acad. Sci. Paris Sér. I Math. 300 (1985), 677-680.

6. A. Connes, J. Feldman, and B. Weiss, An amenable equivalence relation is generated by a single transformation, Ergodic Theory Dynamical Systems 1 (1981), 431-450.

7. C. Ehresmann, Structures feuilletees, Proc. Fifth Canadian Math. Congress, Univ. Toronto Press, Toronto, 1961, pp. 109-172.

8. D. B. A. Epstein, Transversely hyperbolic 1-dimensional foliations, Asterisque 116 (1984), 53-69.

9. E. Fedida, Sur les feuilletages de Lie, C. R. Acad. Sci. Paris Ser. I Math. 272 (1971), 999-1002.

10. J. Feldman and C. C. Moore, Ergodic equivalence relations, cohomology, and von Neumann algebras. I, Trans. Amer. Math. Soc. 234 (1977), 289-324.

11. J. Feldman, P. Hahn and C. C. Moore, Orbit structure and countable sections for actions of continuous groups, Adv. in Math. 28 (1978), 186-230.

12. E. Ghys, Groupes d'holonomie des feuilletages de Lie, Nederl. Akad. Wetensch. Proc. Ser. A 88 (1985), 173-182.

13. A. Haefliger, Structures feuilletees et cohomologie a valeur dans un faisceau de groupoides, Comment. Math. Helv. 32 (1958), 248-329.

14. B. Kostant, On existence and irreducibility of certain series of representations, Bull. Amer. Math. Soc. 75 (1969), 627-642.

15. G. A. Margulis, Discrete groups of motions of manifolds of non-positive curvature, Amer. Math. Soc. Transl. 109 (1977), 33-45.

16. __ Finiteness of quotient groups of discrete subgroups, Functional Anal. Appl. 13 (1979), 178-187.

17. P. Molino, Geometrie globale des feuilletages riemanniens, Nederl. Akad. Wetensch. Proc. Ser. A 85 (1982), 45-76.

18. C. C. Moore, Amenable subgroups of semisimple groups and proximal flows, Israel J. Math. 34 (1979), 121-138.

19. M. S. Raghunathan, Discrete subgroups of Lie groups, Springer-Verlag, New York, 1972.

20. A. Ramsay, Virtual groups and group actions, Adv. in Math. 6 (1971), 253-322.

21. G. Reeb, Sur certains proprietes topologiques des varietes feuilletees, Actualités Sci. Indust, Hermann, Paris, 1952.

22. B. Reinhart, Foliated manifolds with bundle-like metrics, Ann. of Math. (2) 69 (1959), 119 132.

23. K. Schmidt, Cocycles of ergodic transformation groups, MacMillan, India, 1977.

24. W. Thurston, The geometry and topology of 3-manifolds, Princeton Univ. lecture notes.

25. R. J. Zimmer, Amenable ergodic group actions and an application to Poisson boundaries of random walks, J. Funct. Anal. 27 (1978), 350-372.

26. __ Strong rigidity for ergodic actions of semisimple Lie groups, Ann. of Math. (2) 112 (1980), 511-529.

27. __ Orbit equivalence and rigidity of ergodic actions of Lie groups, Ergodic Theory Dynamical Systems 1 (1981), 237-253.

28. __ Ergodic theory and semisimple groups, Birkhauser, Boston, 1984.

29. __, Kazhdan groups acting on compact manifolds, Invent. Math. 75 (1984), 425-436.

30. _ Ergodic theory and the automorphism group of a G-structure (Proc. Conf. in honor of G. W. Mackey, Berkeley, 1984), M.S.R.I. Publications, Springer, 1987.

31 _ _ Amenable actions and dense subgroups of Lie groups, J. Funct. Anal. 27 (1987), 58-64. 